\title{
Auxiliary Atomic Relay Center Facilitates Enhanced Magnetic Couplings in Blatter's Radical
}

\author{
Ashima Bajaj, ${ }^{\dagger}, \ddagger$ Rishu Khurana, ${ }^{\dagger, \ddagger}$ and Md. Ehesan Ali ${ }^{*, \dagger}$ \\ $\dagger$ Institute of Nano Science and Technology, Sector-81, Mohali, Punjab-140306, India \\ $\$$ These authors contributed equally to this work \\ E-mail: ehesan.ali@inst.ac.in
}




\begin{abstract}
The recent accomplishments in obtaining the strong ferromagnetic exchange interactions in organic diradicals have made the field quite fascinating and even more promising towards its technological applications. In this context, herein we report a unique combination of remarkably strong ferromagnetic exchange interactions coupled with the molecular rigidity utilizing super-stable Blatter's radical as a spin source. The planar analogues of the parent Blatter's radical obtained by annulation with a chalcogen coupled to nitronyl nitroxide (NN) are investigated using density functional theory (DFT) along with the wave function based multi-configurational self-consistent field (MCSCF) methods e.g. CASSCF/NEVPT2. The calculations reveal phenomenal modulation in exchange couplings upon annulation such that remarkably strong ferromagnetic interactions are realized especially for a certain class of the Blatter nitronyl nitroxide diradicals. The modulation of spin-spin interactions is rationalised by variation in spin density distribution and molecular torsional angles. We demonstrate that annulation in OMMs opens an additional coupling pathway via auxiliary $\mathrm{X}$-atom acting as atomic relay center which strongly manipulates the magnitude of exchange couplings.
\end{abstract}

\title{
1 Introduction
}

A key step in advancing the organic magnetic architecture towards technological viability is the rational design and synthesis of the exotic molecules with high spin ground states exhibiting strong ferromagnetic interactions. ${ }^{1}$ Such high spin molecules possess extensive applications in high density spin-based memory storage devices and processing units. ${ }^{2,3}$ Diradicals have always remained intrinsically interesting for organic community in which the parallel and antiparallel alignment of two unpaired spins give rise to atleast two states, i.e., a high spin (triplet) and low spin (singlet) respectively. ${ }^{4,5}$ A plethora of literature is replete with the applications of triplet ground state diradicals . ${ }^{6,7}$ However, the room temperature 
stability of triplet ground state diradical, a necessary prerequisite for material processing and device fabrication, has remained highly rare and uncommon and thus has become a fascinating challenge for researchers. With this ongoing challenge, 1,2,4-benzotriazinyl (Blatter) radical emerged out as a promising candidate to develop novel high spin organic magnetic molecules possessing remarkable thermal stability. ${ }^{8-10}$ The radical was first synthesized by Blatter and co-workers in $1968^{11}$ and later several synthetic strategies were introduced by Koutentis et al. over the last few years for the easy synthesis of the super stable Blatter's radical. ${ }^{12,13}$

With a goal to generate stable triplet molecule, Wudl and coworkers presented the first synthesis and characterization of Blatter's based kekule type diradicals. However, contrary to the expectations, zwitterionic forms and open-shell singlets with intermolecular quintet state were obtained. ${ }^{14}$ A milestone was then achieved by Rajca et al. in 2016 with the demonstration of thermally robust triplet ground state diradical by appending nitronyl nitroxide $(\mathrm{NN})$ radical to Blatter's radical with high singlet-triplet splitting. ${ }^{15}$ Triggered by this, we modeled several ferromagnetically coupled hybrid Blatter's based diradicals in our previous works. Employing state-of-art multireference (CASSCF-NEVPT2) methods, quite strong exchange couplings were predicted by attaching several stable radical moieties including oxoverdazyl (OVER), dithiadiazolyl (DTDA), imino nitroxide (IN), nitroxy (NO) and phenoxy (PO) to the stable Blatter's radical. ${ }^{16}$ Apart from the hybrid diradicals, we also considered different possibilities to couple two Blatter's radical with each other and realized that contrary to the hybrid diradicals, it is quite challenging to obtain strong ferromagnetic exchange interactions in di-Blatter diradicals due to co-existing multiple exchange interactions between the several localized spin centers. ${ }^{17}$

Fig. 1a and 1b shows the basic structure and spin density distribution of Blatter's radical. Apart from the spin bearing triazinyl ring (marked as A) containing $3 \mathrm{~N}$-atoms, the radical possess three functional aromatic rings (marked as B, C and D in Fig. 1a). Ring D exhibits negligible spin density and is thus, spin isolated. Hence, it cannot affect the 
magnetic properties significantly. Ring C possesses small delocalization tails of the unpaired electron essentially localized on the triazinyl ring (ring A) whereas ring B contains the major proportion of the unpaired electron. This extensive unpaired spin delocalization over the molecular frame increases the possibility of tuning the magnetic coupling through numerous pathways mediated via ring B and ring C. The diradical proposed by Rajca et al. in 2016 by coupling Blatter's radical with NN radical and exploiting ring $\mathrm{C}$ as a coupler yields magnetic exchange coupling of $174.80 \mathrm{~cm}^{-1} \cdot{ }^{15}$ Later in 2019, instead of ring C, ring B was used as a coupler for spin spin interactions and extremely strong exchange coupling constant of 608 $\mathrm{cm}^{-1}$ was realized by them. ${ }^{18}$ Further, in our previous work, we utilized ring $\mathrm{C}$ as a coupler for modelling hybrid Blatter's diradicals and demonstrated that exchange interactions mediated by ring $\mathrm{C}$ can be further tuned by delocalizing the spin density on ring $\mathrm{B}$ by attaching additional fused benzene rings. ${ }^{16}$

(a)

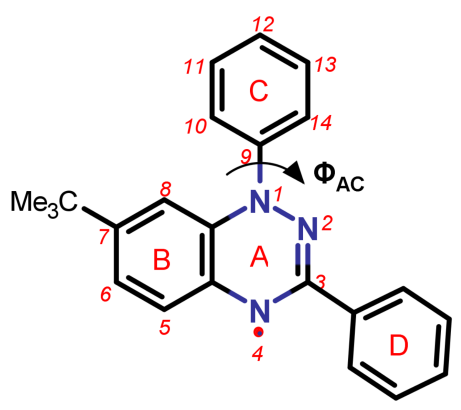

(c)

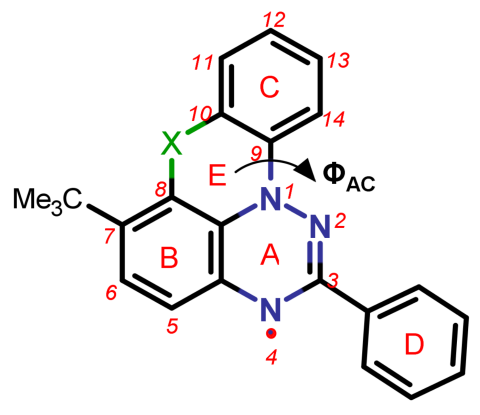

(b)

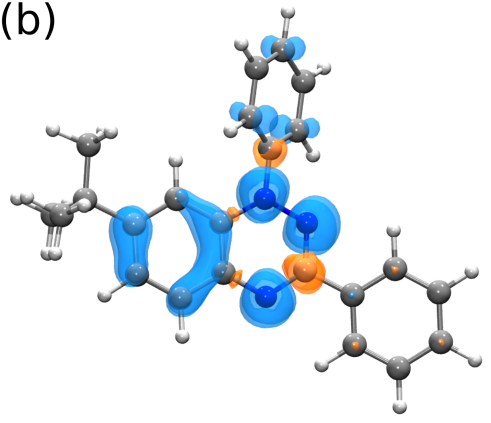

(d)

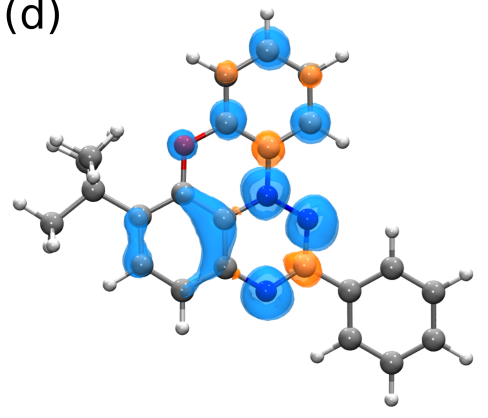

Figure 1: (a) 1,2,4-benzotriazinyl (Blatter's) radical with triazinyl ring containing $3 \mathrm{~N}$ atoms (marked as A) and three aromatic rings marked as $\mathrm{B}, \mathrm{C}$ and $\mathrm{D}, \phi_{A C}$ is the dihedral angle between ring A and ring C. (b) Spin density distribution where blue and orange color represents $\alpha$ and $\beta$ spin respectively with an isovalue of 0.002 (c) Planar analogue of Blatter's radical obtained by annulation at $\mathrm{C} 8$ and $\mathrm{C} 10$ position with $\mathbf{X}(\mathrm{X}=\mathrm{O}, \mathrm{S}$ and $\mathrm{Se})$. (d) Spin density distribution for $\mathrm{X}=\mathrm{O}$ case. 
With this understanding, here in this work we envision a tantalizing prospect to reach even higher values of $2 J$ with simultaneous rigidification of molecular structure by employing planarized analogue of prototypical Blatter's radical shown in Fig. 1c. The planar counterparts are attained by peri-annulation accomplished with the connection of $\mathrm{C}(8)$ and $\mathrm{C}(10)$ positions with a chalcogen $(\mathrm{X}=\mathrm{O}, \mathrm{S}$ and $\mathrm{Se})$ called here as $\mathrm{X}$-annulation. We demonstrate that the co-planar $\mathrm{X}$-annulation opens a new coupling pathway which act as magnetic relay between the spin centers and manipulates the direction of exchange-dominated spin waves in such a way that it promotes remarkably large ferromagnetic interactions. The modulation of exchange coupling upon planarization of Blatter's radical has been explored using both ring $\mathrm{B}$ (called here as Class B diradicals) and ring $\mathrm{C}$ (called here as Class C diradicals) as a coupler which mediates the exchange interactions between spin centers. The computations of intramolecular magnetic exchange coupling is indeed a challenging task and strides of sophisticated theoretical techniques have been proposed so far. ${ }^{19,20}$ In the current work, apart from the traditional DFT based broken symmetry methods, we have adopted spin-constraint DFT (CBS-DFT) to compute the exchange couplings. Further, a mandatory course to multireference methods is performed using symmetry adopted methods such as CASSCF and CASSCF-NEVPT2 to achieve an appropriate quantitative description of the exchange couplings.

\section{Computational Methodology}

The magnetic exchange interactions $(2 J)$ between the two magnetic sites A and B is expressed by phenomenological Heisenberg-Dirac-van Vleck $(H D v V)$ spin Hamiltonian as $\hat{H}_{H D v V}=$ $-2 J \hat{S}_{A} \hat{S}_{B}$, where $\hat{S}_{A}\left(\hat{S}_{B}\right)$ are the local spin operators acting on the sites A (B) and $2 J$ is the effective exchange coupling constant between the radical sites which is equivalent to singlet-triplet energy splitting for a diradical written as 


$$
E_{S}-E_{T}=2 J
$$

where $E_{\mathrm{S}}\left(E_{\mathrm{T}}\right)$ are the energies of singlet (triplet) spin states. A positive value of $2 J$ is representative of ferromagnetic interactions with a triplet ground state whereas a negative value favors anti-ferromagnetic alignment of spins. Computationally, the multi-determinantal nature of the low-spin state wavefunction renders the conventional single-reference (UDFT/UHF) methods unsuitable for modeling the low spin state and thus, multireference techniques (MCSCF/CAS-CI) are often required. An alternative way to evaluate $2 J$ is the broken symmetry (BS) approach within the DFT framework wherein the $2 J$ values are extracted using Noodleman's ${ }^{21}$ spin-projected broken symmetry expression,

$$
2 J=2\left(E_{B S}-E_{T}\right) / S_{\text {max }}^{2},
$$

where $E_{\mathrm{BS}}$ is the energy of broken-symmetry state. While owing to its computational viability, BS-DFT has always been at the forefront and is routinely employed for predicting the exchange couplings, however it is plagued with several deficiencies. ${ }^{22-24}$ The varying degrees of self-interaction errors associated with the traditional DFT functionals results in the spurious delocalization of the magnetic orbitals and hence over predicted $2 J$ values. ${ }^{25,26}$ To mitigate these prevailing shortcomings, spin-constraint DFT (CBS-DFT) appears as a promising approach in which molecule is subjected to spin density constraints within the specified spatial zones. ${ }^{27,28}$ The total energies $E_{\mathrm{BS}}$ and $E_{\mathrm{HS}}$ required for evaluating $2 J$ (using eq. 2) are then computed by applying spin density constraints independently on BS and HS determinants. ${ }^{29}$ The detailed criteria employed for zone selection is discussed in SI. All the BS-DFT and wave-function based calculations are performed in ORCA, ${ }^{30}$ while NWChem ${ }^{31}$ was employed for CBS-DFT calculations.

For all the radical species under study, geometry optimizations are performed using UB3LYP/def2-TZVP method. ${ }^{32}$ Apart from the aforementioned density based approaches, 
the energy eigenvalues of the singlet and triplet states are computed applying multi-configurational wave-function based complete active space self-consistent field (CASSCF) method wherein the $2 J$ values are obtained using Eq. $1 .{ }^{33,34}$ The corresponding CASSCF wavefunction is used as a reference unperturbed wavefunction for subsequent N-electron valence state perturbation theory (NEVPT2) calculations for the treatment of dynamical correlation. ${ }^{35}$ As a general rule of thumb, a natural choice of the CAS space for diradicals is the minimal and the most tractable $\mathrm{CAS}(2,2)$ space, which is obtained by distributing one electron in each singly occupied molecular orbital (SOMO). ${ }^{36}$ For all the diradicals under study, the sign of exchange coupling is well reproduced even at the minimal CAS level, but expectedly the effect of dynamical correlation is not negligible at all. ${ }^{37,38} \mathrm{CAS}(2,2)$ results in underestimated exchange couplings, while considering $\mathrm{CAS}(2,2)$ as a reference wave-function, NEVPT2 increases the absolute $2 J$ value by a factor of $\sim 1.5$, thus reproducing the exchange couplings in close agreement to the experimental ones. The minimal $\operatorname{CAS}(2,2)$ space along with the inclusion of dynamic correlation by NEVPT2 has also shown to be a reasonable choice for Blatter's based diradicals at an acceptable computational cost in our previous works. ${ }^{16,17}$ Here, the CASSCF calculations are further benchmarked for X-annulated counterparts by extending the CAS space upto CAS $(10,10)$ with the inclusion of $\pi$ orbitals of coupler ring and radical centers. The calculated $2 J$ values and the character of the included active orbitals are schematically shown in SI. Although expanding the CAS space improves the static correlations for most of the cases, however, CAS $(10,10)$ results in an uncertain perturbative estimation for $\mathrm{X}=\mathrm{S}$ and $\mathrm{X}=$ Se case. While for $\mathrm{X}=\mathrm{O}$ case, $\mathrm{CAS}(2,2)+\mathrm{NEVPT} 2$ and CAS $(10,10)+$ NEVPT2 produces $2 J$ values with only a marginable difference. Thus, making evident the use of computationally economical minimal $\operatorname{CAS}(2,2)$ space for the reliable estimation of exchange couplings. 


\section{Results and Discussions}

\subsection{X-annulation in Blatter's monoradical}

As a general feature of parent Blatter's radical (shown in Fig. 1a), the steric interactions between $\mathrm{C} 8-\mathrm{H}$ and $\mathrm{C} 10-\mathrm{H}$ result in a large dihedral angle between ring $\mathrm{A}$ and ring $\mathrm{C}$ with $\phi_{A C}=54.02^{\circ}$ (tabulated in Table 1 ). This high torsional angle between the $\pi$ rings inhibits the delocalization of spin density from the spin bearing triazinyl ring to the aromatic ring $\mathrm{C}$ (spin density plot shown in Fig. 1b). One of the possible remedy to this inhibition could be a coplanar $\pi$ substituent at N1 which can promote maximum spin delocalization at ring $\mathrm{C}$ and thus can significantly affect the magnetic properties. In this light, Kaszynski et al. demonstrated the concept of planar Blatter's radical with the synthesis of several functionalized derivatives of planar Blatter's radical by connecting $\mathrm{C}(8)$ and $\mathrm{C}(10)$ positions with oxygen and sulphur annulation. ${ }^{39-41}$ As tabulated in last column of Table 1, O-annulation results in a much smaller dihedral angle of $\phi_{A C}=3.04^{\circ}$, thus approaching the completely planar geometry. However, owing to the bulkiness, $\mathrm{X}=\mathrm{S}$ and Se results in moderate dihedral angles of $20.54^{\circ}$ and $29.96^{\circ}$ respectively. The DFT based löwdin spin populations computed at B3LYP/def2-TZVP level and summed up over the individual aromatic rings of the Blatter's radical listed in Table 1 unambiguously confirms the modification in the spin density distribution brought about by X-annulation. The decrease in spin density on ring $\mathrm{A}$ and ring $\mathrm{B}$ for $\mathrm{X}-\mathrm{Bl}$ as compared to parent Blatter $(\mathrm{Bl})$ reveals that upon $\mathrm{X}$-annulation, the spin density is drained from ring $\mathrm{A}$ and $\mathrm{B}$ towards ring $\mathrm{C}$ in such a way that it results in a transfer of total $10.5 \%$ of spin density from benzotriazinyl core (ring $\mathrm{A}+\operatorname{ring} \mathrm{B}$ ) to ring $\mathrm{C}$ upon O-annulation. Similarly for S-Bl and Se-Bl, ring annulation results in a net transfer of $8.6 \%$ and $6.6 \%$ respectively from benzotriazinyl core to ring C. This consequently leads to an increase in the spin density on the ring $\mathrm{C}$ from 0.058 in $\mathrm{Bl}$ to 0.132 (plus $\rho_{\mathrm{O}}=0.029$ ) in $\mathrm{O}-\mathrm{Bl}$. Followed by this, $\mathrm{S}-\mathrm{Bl}$ and $\mathrm{Se}-\mathrm{Bl}$ also yields enhanced spin delocalization over ring 
$\mathrm{C}$ with a spin density of 0.111 (plus $\rho_{\mathrm{S}}=0.030$ ) and 0.100 (plus $\rho_{\mathrm{Se}}=0.021$ ) respectively. The enhanced spin delocalization over ring $\mathrm{C}$ in $\mathrm{X}-\mathrm{Bl}$ as compared to parent $\mathrm{Bl}$ can also be evidently observed from the spin density plot of $\mathrm{Bl}$ and $\mathrm{O}-\mathrm{Bl}$ shown in Fig. 1c and Fig. 1d respectively.

Table 1: Löwdin spin-density on the individual aromatic rings $(\mathrm{A}, \mathrm{B}, \mathrm{C}$ and D) and dihedral angle between ring $\mathrm{A}$ and ring $\mathrm{C}\left(\phi_{A C}\right)$ of Blatter's radical and $\mathrm{X}$-annulated counterparts with $\mathrm{X}=\mathrm{O}, \mathrm{S}$ and Se using B3LYP/def2-TZVP method.

\begin{tabular}{cccccc}
\hline \multirow{2}{*}{ Radical } & \multicolumn{5}{c}{$\sum \mu($ Ring $)$} \\
\cline { 2 - 5 } & Ring A & Ring B & Ring C & Ring D & \\
\hline Bl & 0.791 & 0.250 & 0.058 & -0.015 & 54.02 \\
O-Bl & 0.750 & 0.203 & 0.132 & -0.010 & 3.04 \\
S-Bl & 0.752 & 0.214 & 0.111 & -0.012 & 20.54 \\
Se-Bl & 0.758 & 0.223 & 0.100 & -0.014 & 29.96 \\
\hline
\end{tabular}

\subsection{Magnetic Exchange Interactions in X-annulated Blatter's Di- radicals}

After gaining insights into the redistribution of spin density within Blatter's radical upon $\mathrm{X}$-annulation, an intriguing quest arises for how the redistribution of spin density coupled with the rigid architecture provided by the X-annulation can affect the existing ferromagnetic interactions in Blatter's based diradicals. The novel Blatter-NN diradical (referred here as Bl-NN) experimentally reported by Rajca et al. ${ }^{15,18}$ and theoretically investigated in our previous work ${ }^{16}$ provides an ideal testing ground for $\mathrm{X}$-annulated architecture. Thus, the model X-annulated systems are conceptualized based on nitronyl nitroxide (NN) as a spin center attached to Blatter's radical with X-annulation. Further, depending upon the coupler ring, diradicals in this work have been divided into two distinct classes, the one in which ring C of Blatter's radical is used as a coupler to connect NN (shown in Fig. 2a) are described as "Class C" and the other which are coupled via ring B (shown in Fig. 2d) are described as "Class B". 


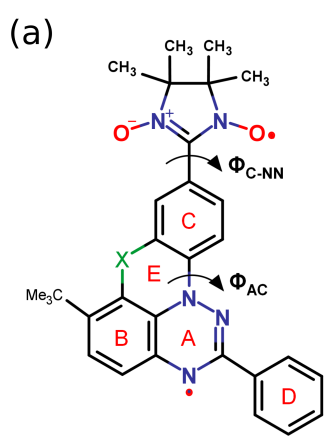

(d)

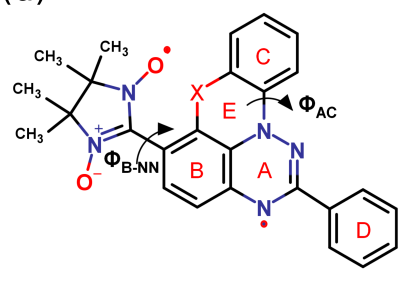

(b)

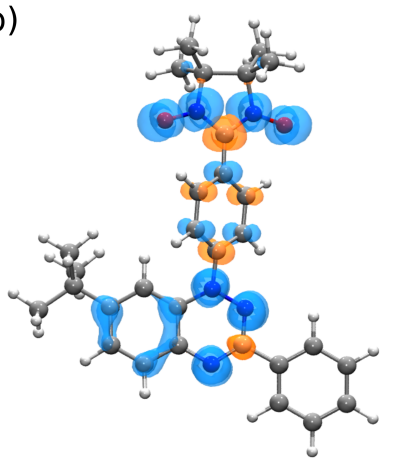

(e)

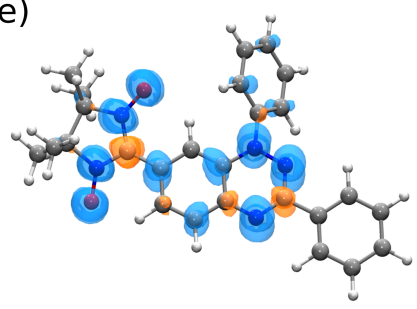

(c)

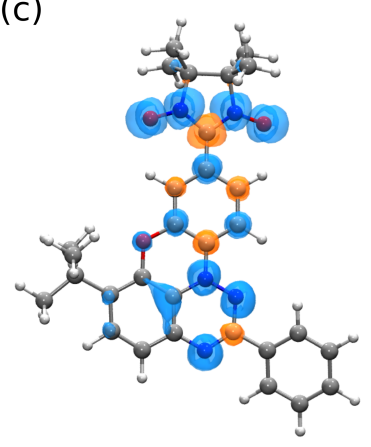

(f)

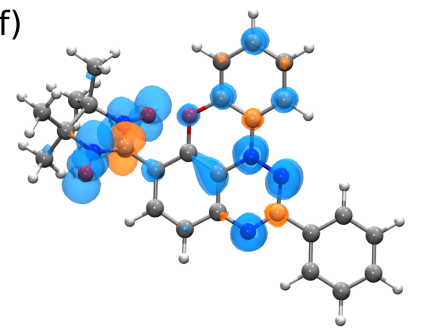

Figure 2: X-annulated Blatter's radical coupled to nitronyl nitroxide (NN) (a) Class C coupled via ring C, X-Bl-NN(C). (b-c) Spin density distribution for Bl-NN(C) and OBl-NN(C) where blue and orange color represents $\alpha$ and $\beta$ spin respectively with an isovalue of 0.003. (d) Class B, X-Bl-NN(B) coupled via ring B. (e-f) Spin density distribution for $\mathrm{Bl}-\mathrm{NN}(\mathrm{B})$ and $\mathrm{O}-\mathrm{Bl}-\mathrm{NN}(\mathrm{B})$.

The computed exchange interactions for Bl-NN diradicals and the X-annulated counterparts are collected in Table 2. Among the Class C diradicals (listed in the top panel of Table 2), the complete method standardization for determining the strength of exchange coupling constant was performed for Bl-NN(C) in our previous work. ${ }^{16}$ Within the traditional broken symmetry approach, even the hybrid B3LYP functional provides an overestimated exchange coupling of $397.80 \mathrm{~cm}^{-1}$. Although this overestimation of exchange coupling by BS-DFT is well known, even though it is the most widely used and arguably the most effective computational tool currently available for the qualitative understanding of the exchange interactions among the spin carriers. ${ }^{42,43}$ Further, in the light of above stated deficiencies of BS-DFT, we adopted an alternative constrained DFT (CBS-DFT) method which dramatically improves the agreement with experiment by providing $2 J$ value of $219.47 \mathrm{~cm}^{-1}$. Apart from this, a more physical treatment of low-spin state requires multi-reference treatment based on CASSCF. We found that the minimal CAS $(2,2)$ space, taking into account 
two unpaired electrons in two SOMOs, provides a $2 J$ value of $96.56 \mathrm{~cm}^{-1}$, a factor of 1.85 smaller than the experimentally reported value. However, adding post SCF correlation by NEVPT2 significantly improves the value to $177.77 \mathrm{~cm}^{-1}$, which is in close agreement to the experimental value of $174.80 \mathrm{~cm}^{-1}$ obtained using SQUID magnetometry. ${ }^{15}$

Later in 2019, Rajca et al. reported another Bl-NN derivative by utilizing the co-planar ring $\mathrm{B}$ as a coupler between the triazinyl ring and $\mathrm{NN}$ radical (called here as $\mathrm{Bl}-\mathrm{NN}(\mathrm{B})$ ), with significantly strong exchange coupling of $608.50 \mathrm{~cm}^{-1}$. Surprisingly, for Bl-NN(B), along with BS-DFT, the more reliable CASSCF $(2,2)+\mathrm{NEVPT} 2$ also results in slightly overestimated exchange coupling by producing the $2 J$ value of $919.48 \mathrm{~cm}^{-1}$ as compared to the experimentally reported value of $608.50 \mathrm{~cm}^{-1} .{ }^{18}$ However, the source of this ambiguity was predicted as mainly due to different dihedral angle $\phi_{A C}$ obtained from the crystal geometry, which is known to have a non-negligible effect on the exchange coupling. ${ }^{44,45}$ The crystal structure yields smaller $\phi_{A C}=39.07^{\circ}$ as compared to B3LYP/def2-TZVP geometry exhibiting $\phi_{A C}=51.40^{\circ}$. As already discussed for Blatter's radical, the smaller $\phi_{A C}$ promotes spin delocalization over ring $\mathrm{C}$ by draining the spin density from benzotriazinyl core (ring $\mathrm{A}+$ ring $\mathrm{B}$ ) to ring $\mathrm{C}$. The reduced spin density on the ring B decreases the strength of spin spin interactions mediated by ring B used as a coupler. This fact is reflected in all the DFT and wave-function based calculations which produces smaller $2 J$ values on crystal structure as compared to DFT optimized geometry (Data for calculation on crystal structure is given in SI). For the further authenticity of B3LYP/def2-TZVP optimized geometry, frequency calculations were also performed at the same level of theory to confirm whether the optimized geometry is true minima of potential energy surface, wherein no any imaginary frequencies were obtained. As an another veracity test, geometry optimization of Bl-NN(B) was also performed with another hybrid M06 functional which also yields a dihedral angle $\phi_{A C}=49.40^{\circ}$ resembling closely to B3LYP optimized geometry. Thus, the source of this geometrical mismatch can be attributed to intrinsic nature of the crystal and environmental effects for which the CASSCF $(2,2)+$ NEVPT2 calculations on the crystal geometry yields a better $2 J$ value 
of $784.05 \mathrm{~cm}^{-1}$. Apart from the geometrical difference, extended CAS calculations on both the crystal and optimized geometry (given in SI) reveals further sequential improvement in dynamical correlation with increasing CAS space. The closest agreement with the reference value of $608.50 \mathrm{~cm}^{-1}$ is obtained with NEVPT2 based on extended CAS $(10,10)$ producing $2 J$ value of $659.81 \mathrm{~cm}^{-1}$ at crystal geometry. The apparent low performance of $\mathrm{CAS}(2,2)$ space to deal with Bl-NN(B) is attributed to the SOMOs having node on the bridging C(sp2) atom of O-N-C-N-O fragment of NN moeity and thus does not taking into account the spin polarization effects on the bridging atoms which are more prominent in Class B due to more spin dense connecting site C7 in Class B as compared to C12 in Class C diradicals. The role of bridging $\mathrm{C}$-atom of $\mathrm{O}-\mathrm{N}-\mathrm{C}-\mathrm{N}-\mathrm{O}$ fragment of $\mathrm{NN}$ radical in the reference $\mathrm{CAS}$ space was also demonstrated in an early report by Calzado et al. ${ }^{37}$

Table 2: Calculated magnetic exchange coupling constants for Class B and Class C Bl-NN diradicals with X-annulation using BS-DFT, CBS-DFT, CASSCF $(2,2)$ and CASSCF $(2,2)-N E V P T 2$.

\begin{tabular}{cccccc}
\hline Diradicals & \multicolumn{5}{c}{$2 J\left(\mathrm{~cm}^{-1}\right)$} \\
\cline { 2 - 6 } & BS-DFT & CBS-DFT & CASSCF $(2,2)$ & CASSCF $(2,2)+$ NEVPT2 & Exp. \\
\hline Bl-NN(C) & 397.80 & 219.47 & 96.56 & 177.77 & 174.80 \\
O-Bl-NN(C) & 1132.54 & 615.03 & 324.50 & 595.32 & - \\
S-Bl-NN(C) & 893.98 & 489.92 & 241.11 & 454.52 & - \\
Se-Bl-NN(C) & 824.76 & 453.85 & 217.35 & 416.02 & - \\
\hline Bl-NN(B) & 1216.78 & 644.45 & 521.97 & 919.48 & 608.50 \\
O-Bl-NN(B) & 280.60 & 151.89 & 152.55 & 217.30 & - \\
S-Bl-NN(B) & 632.88 & 350.32 & 327.05 & 528.55 & - \\
Se-Bl-NN(B) & 716.54 & 393.56 & 351.85 & 617.67 & - \\
\hline
\end{tabular}

${ }^{a}$ For Bl-NN(B), calculations on crystal geometry yields $2 J$ value of $784.05 \mathrm{~cm}^{-1}$ using CASSCF $(2,2)+$ NEVPT2.

Following the same recipe for both Class C and Class B X-annulated diradicals, we observed that X-annulation significantly effects the degree of exchange coupling. Interestingly, for Class $\mathrm{C}$ diradicals, $\mathrm{X}$-annulation resulted in remarkably strong ferromagnetic exchange coupling with an appreciable increase in $2 J$ values by a factor of 2 to 3 as compared to Bl$\mathrm{NN}(\mathrm{C})$. Among the three chalcogens, O-congener produced the strongest exchange coupling for which the $2 J$ value increases upon O-annulation by a factor of 2.85 with BS-DFT and the 
most promising $\operatorname{CASSCF}(2,2)+\mathrm{NEVPT} 2$ also reveals a significant enhancement in $2 J$ value from $177.77 \mathrm{~cm}^{-1}$ for Bl-NN(C) to $595.32 \mathrm{~cm}^{-1}$ for O-Bl-NN(C). An analogous increase is also observed for $\mathrm{S}$ and Se analogs in the series with increased $2 J$ values of $454.52 \mathrm{~cm}^{-1}$ and $416.02 \mathrm{~cm}^{-1}$ respectively. While in complete contrast to Class C, Class B diradicals (listed in bottom panel of Table 2) displays a drastic decrease in $2 J$ values upon X-annulation, with the least $2 J$ value being observed for $\mathrm{X}=\mathrm{O}$. For O-Bl-NN(B), both BS-DFT as well as $\operatorname{CASSCF}(2,2)+$ NEVPT2 results in a drop of $2 J$ value by a factor of $\sim 4$ from 919.48 $\mathrm{cm}^{-1}$ for Bl-NN(B) to $217.30 \mathrm{~cm}^{-1}$ for O-Bl-NN(B). The similar decrease in $2 J$ values also manifests in S-Bl-NN(B) and Se-Bl-NN(B), but with slightly larger exchange couplings as compared to $\mathrm{O}-\mathrm{Bl}-\mathrm{NN}(\mathrm{B})$. The substantial variation in $2 J$ values brought upon $\mathrm{X}$-annulation is also shown in Fig. 3 for both Class B and Class C.

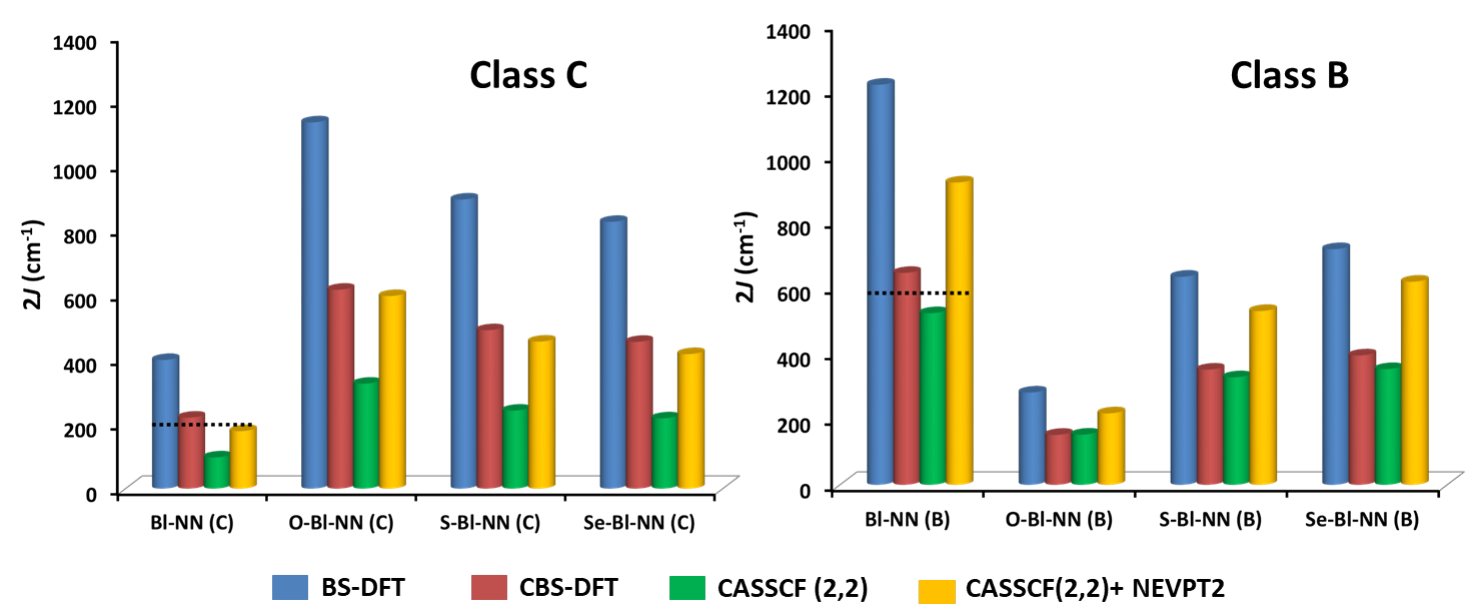

Figure 3: Variation in magnetic exchange coupling $(2 J)$ for X-annulated Class C (left) and Class B (right) Bl-NN diradicals. Blue, red, green and yellow bars indicate the calculated exchange coupling constants using BS-DFT, CBS-DFT, CASSCF $(2,2)$ and CASSCF $(2,2)+\mathrm{NEVPT} 2$ respectively. The black dotted line over Bl-NN(C) and Bl-NN(B) represents the experimental $2 J$ value. For Class $\mathrm{C}, 2 J$ values increases upon X-annulation and vice-versa for Class B. 


\subsection{Modification in Spin Density Distribution and Torsional An- gles}

As already discussed for monoradicals, the salient electronic feature of $\mathrm{X}$-annulated counterparts is the nearly planar geometry and enhanced spin delocalization over the ring $\mathrm{C}$. Gratifyingly, this characteristic feature promotes the spin spin interactions for Class C and vice-versa the case for Class B which is reflected in the form of enhanced exchange couplings for Class $\mathrm{C}$ and reduced ones for Class B. The spin density distribution for Class C and Class B Bl-NN diradicals and O-annulated counterparts shown in Fig. 2 and tabulated in Table 3 reveals the anticipated modification in the spin distribution upon X-annulation. For Class $\mathrm{C}$ diradicals, $\mathrm{X}$-annulation results in the reduction of spin density on the benzotriazinyl core wherein for ring A, it decreases from 0.765 for Bl-NN(C) to 0.709, 0.720 and 0.726 and ring $\mathrm{B}$ yields a decrease from 0.245 to $0.192,0.202$ and 0.212 for $\mathrm{X}=\mathrm{O}, \mathrm{S}$ and Se respectively. This overall displacement of the spin density from the benzo-triazinyl core to the ring $\mathrm{C}$ accumulates an additional spin density of $0.06,0.04$ and 0.03 over the ring $\mathrm{C}$ for $\mathrm{X}=\mathrm{O}, \mathrm{S}$ and Se respectively as compared to 0.008 in case of $\mathrm{Bl}-\mathrm{NN}(\mathrm{C})$. The enhanced spin delocalization over ring $\mathrm{C}$ promotes the unidirectional magnetic coupling between spin centers via ring $\mathrm{C}$ which is reflected in the form of increased $2 J$ values. Apart from the modification in spin density, an increased $2 J$ value is also associated with the reduced dihedral angle between the coupler $\mathrm{C}$ and spin carriers, i.e., $\phi_{A C}$ and $\phi_{C-N N}$. For O-Bl-NN(C), $\phi_{A C}$ reduces to nearly zero $\left(4.17^{\circ}\right)$ from a twisted angle of $52.88^{\circ}$ in $\mathrm{Bl}-\mathrm{NN}(\mathrm{C})$. This coplanar coupler-radical center plane further promotes effective spin propagation and vividly support strong exchange interactions. Although, various other approaches in the literature have also used these generalizations based on spin density and dihedral angle for the design of new spin centers, couplers and OMMs, ${ }^{46,47}$ but to our knowledge this is the first report with such a large exchange coupling in Blatter's diradicals coupled with molecular rigidity and nearly planar geometry. Thus, balancing both the stability and the strength of exchange 
interactions, thereby, advancing a step closer towards the practical applications of OMMs.

Table 3: Löwdin spin-density on the individual aromatic rings of Blatter and NN spin sites and dihedral angles computed at B3LYP/def2-TZVP level for both Class $\mathrm{C}$ and Class B Bl-NN diradicals and the X-annulated counterparts.

\begin{tabular}{c|ccccc|cc}
\hline Diradicals & \multicolumn{4}{|c}{$\sum \mu$} \\
\cline { 2 - 7 } & Ring A & Ring B & Ring C & Ring D & NN & $\phi_{A C}$ & $\phi_{C-N N} / \phi_{B-N N}$ \\
\hline Bl-NN(C) & 0.765 & 0.245 & 0.008 & -0.015 & 1.05 & 52.88 & 22.38 \\
O-Bl-NN(C) & 0.709 & 0.192 & 0.068 & -0.011 & 1.08 & 4.17 & 16.30 \\
S-Bl-NN(C) & 0.720 & 0.202 & 0.049 & -0.012 & 1.075 & 20.99 & 20.25 \\
Se-Bl-NN(C) & 0.726 & 0.212 & 0.030 & -0.013 & 1.074 & 28.71 & 16.45 \\
\hline Bl-NN(B) & 0.709 & 0.188 & 0.062 & -0.012 & 1.091 & 51.41 & 20.40 \\
O-Bl-NN(B) & 0.725 & 0.162 & 0.132 & -0.009 & 1.032 & 0.30 & 51.14 \\
S-Bl-NN(B) & 0.703 & 0.167 & 0.110 & -0.012 & 1.059 & 19.75 & 42.27 \\
Se-Bl-NN(B) & 0.706 & 0.170 & 0.102 & -0.012 & 1.06 & 25.86 & 42.87 \\
\hline
\end{tabular}

Similarly, for Class B diradicals, X-annulation impedes the spin delocalization to the ring $\mathrm{C}$ from 0.062 for $\mathrm{Bl}-\mathrm{NN}(\mathrm{B})$ to 0.132 for O-Bl-NN(B) but with a simultaneous decrease in spin density on ring $\mathrm{B}$ which acts as a spin coupling unit for Class B diradicals from 0.188 to 0.162 for Bl-NN(B) and O-Bl-NN(B) respectively. Further, the dihedrals $\phi_{A C}$ and $\phi_{B-N N}$ that describes the extent of planarity and the conjugation between coupler units and two radical centers also reveals an anomalous trend. Similar to Class C, X-annulation results in reduced dihedral angle $\phi_{A C}$, from $51.41^{\circ}$ for $\mathrm{Bl}-\mathrm{NN}(\mathrm{B})$ to $0.30^{\circ}$ for O-Bl-NN(B), thus approaching the planar geometry. However, this is also accompanied by an increase in dihedral angle $\phi_{B-N N}$ from $20.40^{\circ}$ for Bl-NN(B) to $51.41^{\circ}$ for O-Bl-NN(B) due to repulsive interactions between $\mathrm{NN}$ spin center and X-atom attached at the consecutive $\mathrm{C} 7$ and $\mathrm{C} 8$ positions respectively. Thus, the decreased spin delocalizaion over the coupler B and the increased molecular twist $\phi_{B-N N}$ inhibits the spin-spin interactions and results in decreased $2 J$ values for Class B diradicals. Further, to elucidate the origin behind the drastic decrease in $2 J$ value from $1216 \mathrm{~cm}^{-1}$ to $280 \mathrm{~cm}^{-1}$ upon O-annulation in Bl-NN(B) and to investigate the sole effect of O-annulation, we constrained both the dihedral angles $\phi_{A C}$ and $\phi_{B-N N}$ of $\mathrm{Bl}-\mathrm{NN}(\mathrm{B})$ to 0 and $50^{\circ}$ (equivalent to dihedral angles of O-Bl-NN(B)) respectively. The $2 J$ values extracted from BS-DFT calculations on the constrained geometry yields a decreased 
$2 J$ value of $718.52 \mathrm{~cm}^{-1}$ as compared to $1216 \mathrm{~cm}^{-1}$ extracted from the optimized geometry, but significantly higher than $2 J$ value of $280 \mathrm{~cm}^{-1}$ in O-Bl-NN. The spin density analysis over the individual rings of the Blatter's radical in constrained geometry reveals a spin density of 0.279 on the coupler B which reduces to 0.162 upon O-annulation in case of $0-\mathrm{Bl}-\mathrm{NN}(\mathrm{B})$ (tabulated in Table 3). This clearly indicates that apart from the molecular torsional, the secondary channel induced by X-annulation provides additional relay center between spin centers which plays a dominant role in controlling the exchange interactions. Apart from the remarkable modification in exchange couplings brought by X-annulation in Class B and Class C diradicals, we also calculated exchange couplings for Blatter's radical coupled to NN via spin isolated ring D at meta position, i.e., Bl-NN(D) (shown in SI). Although ferromagnetic but quite weak exchange couplings were realized with a negligible effect upon O-annulation (Data in SI).

\subsection{Modeled O-annulated Diradicals}

Furthermore, prompted by an appreciable increase in $2 J$ values for Class C diradicals coupled with molecular rigidity upon O-annulation, here we designed O-annulated counterparts of the modeled Class $\mathrm{C}$ diradicals proposed in our previous work ${ }^{16}$ by connecting stable radicals such as OVER, DTDA and IN to Blatter's radical called here as Bl-OVER(C), Bl-DTDA(C) and $\mathrm{Bl}-\mathrm{IN}(\mathrm{C})$ respectively. The O-annulated analogs of the modeled diradicals labeled as O-Bl-OVER(C), O-Bl-DTDA(C), and O-Bl-IN(C) are shown in Fig. 4 and the respective $2 J$ values are tabulated in Table 4 . 

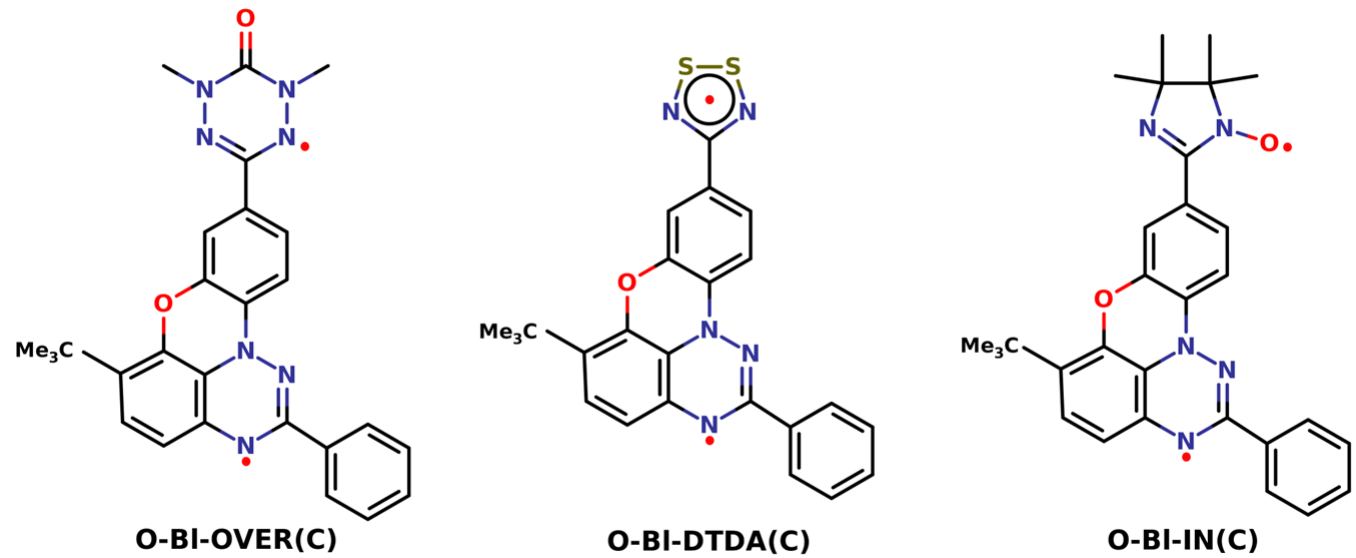

Figure 4: Class $\mathrm{C}$ modelled diradicals with $\mathrm{O}$-annulation by coupling Blatter's radical with OVER, DTDA and IN labeled as O-Bl-OVER(C), O-Bl-DTDA(C) and O-Bl-IN(C) respectively.

As illustrated in Table 4, analogous to Bl-NN(C), all the modeled diradicals yields significant enhancement in the existing ferromagnetic couplings upon O-annulation using all the aforementioned methods. Followed by O-Bl-NN(C), O-Bl-OVER(C) appears as next best candidate exhibiting strong ferromagnetic exchange interactions. However, for OVER based diradicals, the minimal $\mathrm{CAS}(2,2)$ space turns out to be inadequate due to the omitting contribution of the SOMOs from the O-atom of OVER radical. The dynamical correlations on the top of insufficient CAS $(2,2)$ space even bias the results towards anti-ferromagnetic states and lead to incorrect prediction of anti-ferromagnetic ground state, wherein DFT based methods predicts strong ferromagnetic exchange. The balanced treatment of spin states is then obtained by the inclusion of orbitals affiliated with O-atom of OVER radical using CAS $(4,4)$ space. Thus, for all the OVER based diardicals CAS $(4,4)$ space is chosen for determining $2 J$ values. The additional orbitals involved in $\mathrm{CAS}(4,4)$ space along with the SOMOs are presented in SI. Apart from this, the diradical Bl-IN(C) was also synthesized by Rajca et al., ${ }^{15}$ however the authors were not able to quantify the strength of exchange interactions due to weak spin-spin interactions. Interestingly, with O-annulation, O-Bl-IN(C) also yields significantly increased $2 J$ value of $177.98 \mathrm{~cm}^{-1}$ using CASSCF $(2,2)+\operatorname{NEVPT} 2$ with nearly planar geometry exhibiting dihedral angles of $0.96^{\circ}$ and $9.13^{\circ}$ of coupler ring $\mathrm{C}$ with spin 
centers $\mathrm{Bl}$ and IN respectively.

Table 4: Calculated magnetic exchange coupling constants for modeled O-annulated Class C diradicals using BS-DFT, CBS-DFT, CASSCF $(2,2)$, CASSCF(2,2)-NEVPT2.

\begin{tabular}{ccccc}
\hline Diradicals & \multicolumn{3}{c}{$2 J\left(\mathrm{~cm}^{-1}\right)$} \\
\cline { 2 - 5 } & BS-DFT & CBS-DFT & CASSCF $(2,2)$ & CASSCF $(2,2)+$ NEVPT2 \\
\hline Bl-OVER(C) & 312.44 & 254.51 & $118.53^{a}$ & $158.04^{a}$ \\
O-Bl-OVER(C) & 589.76 & 356.73 & $254.40^{a}$ & $325.07^{a}$ \\
\hline Bl-DTDA(C) & 150.38 & 109.73 & 37.31 & 65.84 \\
O-Bl-DTDA(C) & 544.24 & 202.81 & 166.54 & 297.66 \\
\hline Bl-IN(C) & 130.74 & 87.78 & 9.47 & 39.50 \\
O-Bl-IN(C) & 490.92 & 309.49 & 43.56 & 177.98 \\
\hline & \multicolumn{2}{c}{ values are extracted using CAS(4,4) active space. CASSCF $(2,2)$ results are given in }
\end{tabular}

SI.

Similar to Class C modeled diradicals, we also designed a set of ferromagnetically coupled Class B modeled diradicals utilizing ring B as a coupler. Coupling Blatter's radical with OVER, DTDA and IN at $\mathrm{C} 7$ position of ring $\mathrm{B}$ results in quite strong ferromagnetic exchange interactions. However, following the rationale of $\mathrm{Bl}-\mathrm{NN}(\mathrm{B})$, the spin-spin interactions eventually got attenuated upon O-annulation for all the modeled Class B diradicals. The calculated exchange coupling constants for modeled Class B diradicals are provided in SI and the variation of $2 J$ values for both Class B and Class C modeled diradicals in shown in Fig. 5. 


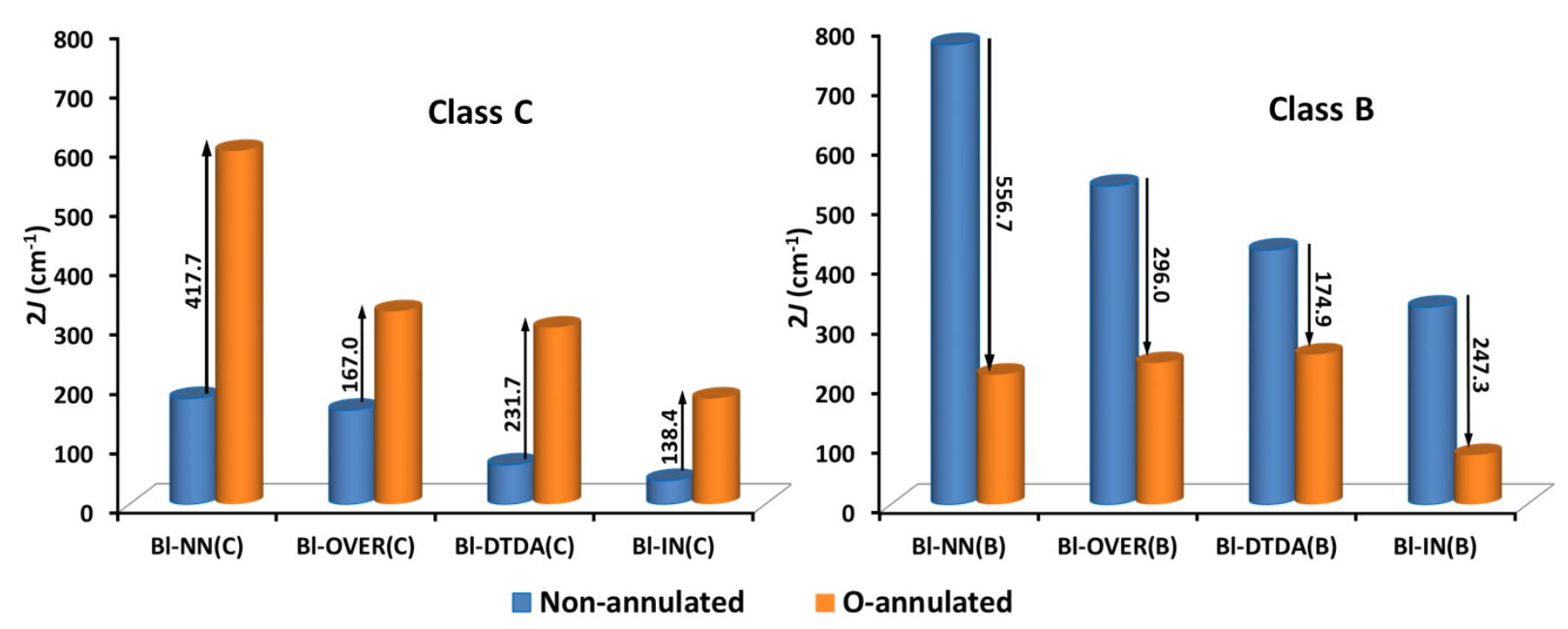

Figure 5: Variation of exchange coupling for modeled Class C (left) and Class B (right) diradicals using CASSCF $(2,2)+$ NEVPT2. The blue and orange bars denotes non-annulated and O-annulated counterparts. For OVER based diradicals, CAS $(4,4)$ space is used. The blue bar for Bl-NN(B) represents the calculated $2 J$ value over crystal geometry.

\section{Conclusion}

In summary, the foregoing results demonstrate the influential role played by X-annulation in controlling the magnetic exchange interactions in Blatter's based diradicals. Two distinct classes of Bl-NN diradical, i.e. Class C and Class B, used here as an optimum testing bed, reveals paradoxical scenario of exchange couplings upon $\mathrm{X}$-annulation. Class $\mathrm{C}$ diradicals results in an appreciable increase in $2 J$ values, while a drastic decrease is observed for Class B diradicals. This remarkable variation in $2 J$ values is rationalised in terms of variation of spin density distribution and molecular torsional angles. For both Class B and Class C, $\mathrm{X}$-annulation results in the enhanced spin delocalization over ring $\mathrm{C}$ at the cost of ring $\mathrm{A}$ and $\mathrm{B}$, which manifests itself in the form of significantly strong exchange couplings reported so far for Class $\mathrm{C}$ diradicals. However, the decrease of spin density over ring $\mathrm{B}$ results in the attenuation of exchange interactions for Class B diradicals. This demonstrates that the secondary channel induced by $\mathrm{X}$-annulation assist the existing exchange interactions between the radical centers via $\mathrm{X}$-atoms acting as relay centers. Thereby providing an example of the secondary pathway assisting the relay like exchange mechanism with an unprecedented 
combination of molecular rigidity and strong ferromagnetic exchange interactions. We propose that the systematic modification of spin density via X-annulation will facilitate the thermally robust and easily synthesizable diradicals with remarkable strong ferromagnetic exchange interactions.

\section{Acknowledgement}

Financial support from Department of Science and Technology through SERB-ECR project No. ECR/2016/000362, Indo-Sweden joint project No. DST/INT/SWD/VR/P-01/2016 is gratefully acknowledged.

Supporting Information Available: Computed total energies and $2 J$ values, selection of the active space for CASSCF/NEVPT2 calculation, Class D Bl-NN diradicals and modeled diradicals.

This material is available free of charge via the Internet at http:// pubs.acs.org.

\section{References}

(1) Lahti, P. M. Adv Phys Org Chem; Elsevier, 2011; Vol. 45; pp 93-169.

(2) Ratera, I.; Veciana, J. Playing with organic radicals as building blocks for functional molecular materials. Chem. Soc. Rev. 2012, 41, 303-349.

(3) Sanvito, S. Molecular spintronics. Chem. Soc. Rev. 2011, 40, 3336-3355.

(4) Abe, M. Diradicals. Chem. Rev. 2013, 113, 7011-7088.

(5) Stuyver, T.; Chen, B.; Zeng, T.; Geerlings, P.; De Proft, F.; Hoffmann, R. Do Diradicals Behave Like Radicals? Chem. Rev. 2019, 119, 11291-11351.

(6) Gallagher, N. M.; Olankitwanit, A.; Rajca, A. High-spin organic molecules. J. Org. Chem. 2015, 80, 1291-1298. 
(7) Lineberger, W. C.; Borden, W. T. The synergy between qualitative theory, quantitative calculations, and direct experiments in understanding, calculating, and measuring the energy differences between the lowest singlet and triplet states of organic diradicals. Phys. Chem. Chem. Phys. 2011, 13, 11792-11813.

(8) Grant, J. A.; Lu, Z.; Tucker, D. E.; Hockin, B. M.; Yufit, D. S.; Fox, M. A.; Kataky, R.; Chechik, V.; O’Donoghue, A. C. New Blatter-type radicals from a bench-stable carbene. Nat. Commun. 2017, 8, 1-6.

(9) Ji, Y.; Long, L.; Zheng, Y. Recent advances of stable Blatter radicals: synthesis, properties and applications. Mater. Chem. Front. 2020, 4, 3433-3443.

(10) Rogers, F.; Norcott, P.; Coote, M. Recent Advances in the Chemistry of Benzo [e][1, 2, 4] triazinyl Radicals. Org. Biomol. Chem. 2020, 18, 8255-8277.

(11) Blatter, H. M.; Lukaszewski, H. A new stable free radical. Tetrahedron Lett. 1968, 9, $2701-2705$.

(12) Savva, A. C.; Mirallai, S. I.; Zissimou, G. A.; Berezin, A. A.; Demetriades, M.; Kourtellaris, A.; Constantinides, C. P.; Nicolaides, C.; Trypiniotis, T.; Koutentis, P. A. Preparation of Blatter Radicals via Aza-Wittig Chemistry: The Reaction of NAryliminophosphoranes with 1-(Het) aroyl-2-aryldiazenes. J. Org. Chem. 2017, 82, 7564-7575.

(13) Constantinides, C. P.; Koutentis, P. A.; Loizou, G. Synthesis of 7-aryl/heteraryl-1, 3-diphenyl-1, 2, 4-benzotriazinyls via palladium catalyzed Stille and Suzuki-Miyaura reactions. Org. Biomol. Chem. 2011, 9, 3122-3125.

(14) Zheng, Y.; Miao, M.-s.; Dantelle, G.; Eisenmenger, N. D.; Wu, G.; Yavuz, I.; Chabinyc, M. L.; Houk, K. N.; Wudl, F. A Solid-State Effect Responsible for an Organic Quintet State at Room Temperature and Ambient Pressure. Adv. Mater. 2015, $27,1718-1723$. 
(15) Gallagher, N. M.; Bauer, J. J.; Pink, M.; Rajca, S.; Rajca, A. High-spin organic diradical with robust stability. J. Am. Chem. Soc. 2016, 138, 9377-9380.

(16) Bajaj, A.; Ali, M. E. First-Principle Design of Blatter's Diradicals with Strong Ferromagnetic Exchange Interactions. J. Phys. Chem. C 2019, 123, 15186-15194.

(17) Khurana, R.; Bajaj, A.; Ali, M. E. How Plausible Is Getting Ferromagnetic Interactions by Coupling Blatter's Radical via Its Fused Benzene Ring? J. Phys. Chem. A 2020, 124, 6707-6713.

(18) Gallagher, N.; Zhang, H.; Junghoefer, T.; Giangrisostomi, E.; Ovsyannikov, R.; Pink, M.; Rajca, S.; Casu, M. B.; Rajca, A. Thermally and magnetically robust triplet ground state diradical. J. Am. Chem. Soc. 2019, 141, 4764-4774.

(19) Sharma, P.; Truhlar, D. G.; Gagliardi, L. Magnetic Coupling in a Tris-hydroxo-Bridged Chromium Dimer Occurs through Ligand Mediated Superexchange in Conjunction with Through-Space Coupling. J. Am. Chem. Soc. 2020, 142, 16644-16650.

(20) Gagliardi, L.; Truhlar, D. G.; Li Manni, G.; Carlson, R. K.; Hoyer, C. E.; Bao, J. L. Multiconfiguration pair-density functional theory: A new way to treat strongly correlated systems. Acc. Chem. Res. 2017, 50, 66-73.

(21) Noodleman, L. Valence bond description of antiferromagnetic coupling in transition metal dimers. J. Chem. Phys. 1981, 74, 5737-5743.

(22) Gräfenstein, J.; Kraka, E.; Filatov, M.; Cremer, D. Can unrestricted density-functional theory describe open shell singlet biradicals? Int. J. Mol. Sci. 2002, 3, 360-394.

(23) Illas, F.; de PR Moreira, I.; Bofill, J.; Filatov, M. Extent and limitations of densityfunctional theory in describing magnetic systems. Phys. Rev. B 2004, 70, 132414.

(24) David, G.; Trinquier, G.; Malrieu, J.-P. Consistent spin decontamination of brokensymmetry calculations of diradicals. J. Chem. Phys. 2020, 153, 194107. 
(25) Mori-Sánchez, P.; Cohen, A. J.; Yang, W. Many-electron self-interaction error in approximate density functionals. J. Chem. Phys. 2006, 125, 201102.

(26) Bao, J. L.; Gagliardi, L.; Truhlar, D. G. Self-interaction error in density functional theory: An appraisal. J. Phys. Chem. Lett. 2018, 9, 2353-2358.

(27) Rudra, I.; Wu, Q.; Van Voorhis, T. Accurate magnetic exchange couplings in transitionmetal complexes from constrained density-functional theory. J. Chem. Phys. 2006, 124, 024103.

(28) Kaduk, B.; Kowalczyk, T.; Van Voorhis, T. Constrained density functional theory. Chem. Rev. 2012, 112, 321-370.

(29) Ali, M. E.; Nair, N. N.; Staemmler, V.; Marx, D. Constrained spin-density dynamics of an iron-sulfur complex: Ferredoxin cofactor. J. Chem. Phys. 2012, 136, 224101.

(30) Neese, F. The ORCA program system. Wiley Interdiscip. Rev. Comput. Mol. Sci. 2012, 2, $73-78$.

(31) Valiev, M.; Bylaska, E. J.; Govind, N.; Kowalski, K.; Straatsma, T. P.; Van Dam, H. J.; Wang, D.; Nieplocha, J.; Apra, E.; Windus, T. L. et al. NWChem: A comprehensive and scalable open-source solution for large scale molecular simulations. Comput. Phys. Commun. 2010, 181, 1477-1489.

(32) Weigend, F.; Ahlrichs, R. Balanced basis sets of split valence, triple zeta valence and quadruple zeta valence quality for H to Rn: Design and assessment of accuracy. Phys. Chem. Chem. Phys. 2005, 7, 3297-3305.

(33) Malrieu, J. P.; Caballol, R.; Calzado, C. J.; de Graaf, C.; Guihery, N. Magnetic interactions in molecules and highly correlated materials: physical content, analytical derivation, and rigorous extraction of magnetic Hamiltonians. Chem. Rev. 2014, 114, $429-492$. 
(34) Khedkar, A.; Roemelt, M. Active space selection based on natural orbital occupation numbers from n-electron valence perturbation theory. J. Chem. Theory Comput. 2019, $15,3522-3536$.

(35) Angeli, C.; Cimiraglia, R.; Evangelisti, S.; Leininger, T.; Malrieu, J.-P. Introduction of n-electron valence states for multireference perturbation theory. J. Chem. Phys. 2001, $114,10252-10264$.

(36) Suaud, N.; Ruamps, R.; Guihery, N.; Malrieu, J.-P. A strategy to determine appropriate active orbitals and accurate magnetic couplings in organic magnetic systems. J. Chem. Theory Comput. 2012, 8, 4127-4137.

(37) Calzado, C. J.; Angeli, C.; de Graaf, C.; Caballol, R. Analysis of the magnetic coupling in nitroxide organic biradicals. Theor. Chem. Acc. 2011, 128, 505-519.

(38) Kaur, P.; Ali, M., et al. First Principle Investigations of Long-range Magnetic Exchange Interactions via Polyacene Coupler. ChemRxiv doi: https://doi.org/10. 26434/chemrxiv.12776222.v1.

(39) Kaszyński, P.; Constantinides, C. P.; Young Jr, V. G. The Planar Blatter Radical: Structural Chemistry of 1, 4-Dihydrobenzo [e][1, 2, 4] triazin-4-yls. Angew. Chem. 2016, 128, 11315-11318.

(40) Bartos, P.; Anand, B.; Pietrzak, A.; Kaszynski, P. Functional Planar Blatter Radical through Pschorr-Type Cyclization. Org. Lett. 2019, 22, 180-184.

(41) Hande, A. A.; Baylere, P.; Kaszynski, P.; Chrostowska, A. Effect of $\pi$-System Extension on the Ionization Energy of the Planar Blatter Radical: Experimental and Theoretical Studies. J. Phys. Chem. A 2020, 124, 9777-9782.

(42) Sadhukhan, T.; Beckett, D.; Thapa, B.; Raghavachari, K. Coupling Constants, High Spin, and Broken Symmetry States of Organic Radicals: an Assessment of the 
Molecules-in-Molecules Fragmentation-Based Method. J. Chem. Theory Comput. 2019, 15, 5998-6009.

(43) Mayhall, N. J.; Head-Gordon, M. Computational Quantum Chemistry for Multiple-Site Heisenberg Spin Couplings Made Simple: Still Only One Spin-Flip Required. J. Phys. Chem. Lett. 2015, 6, 1982-1988.

(44) Ali, M. E.; Datta, S. N. Broken-symmetry density functional theory investigation on bis-nitronyl nitroxide diradicals: influence of length and aromaticity of couplers. $J$. Phys. Chem. A 2006, 110, 2776-2784.

(45) Ali, M. E.; Roy, A. S.; Datta, S. N. Molecular tailoring and prediction of strongly ferromagnetically coupled trimethylenemethane-based nitroxide diradicals. J. Phys. Chem. A 2007, 111, 5523-5527.

(46) Latif, I. A.; Hansda, S.; Datta, S. N. High magnetic exchange coupling constants: a density functional theory based study of substituted Schlenk diradicals. J. Phys. Chem. A 2012, 116, 8599-8607.

(47) Wang, Z.-Y.; Dai, Y.-Z.; Ding, L.; Dong, B.-W.; Jiang, S.-D.; Wang, J.-Y.; Pei, J. A Stable Triplet-Ground-State Conjugated Diradical Based on a Diindenopyrazine Skeleton. Angew. Chem. Int. Ed. 2020, doi: https://doi.org/10.1002/anie.202012989. 